\title{
The Realization of TCP Enhancement Technology in Mobile Wireless Network IP Based
}

\author{
Jianmei Dai \\ Dept. of Informational Equipment \\ Academy of Equipment \\ Beijing, China \\ Benxuelan0402@126.com
}

\author{
Huafang Geng \\ Changping N.C.O School \\ Academy of Equipment \\ Beijing, China \\ Cherry5810@sina.com
}

\begin{abstract}
The transmit control protocol is used more and more widely as the development of mobile wireless network IP based and the need of high reliable data transmission in the unstable environment, but it has a poor performance due to the complex wireless channel and the variable network structure. Two TCP enhancement modules named unilateral TCP-accelerate module and parallel TCP-accelerate module were designed and imported to a test wireless network and a 3G system after analyzing the shortcoming of standard TCP and summarizing the popular TCP enhancement technologies. It proved that the modules designed which are easy to employ and flexible can improve the performance of TCP significant and can be used for reliable and burst transmission of broadband data in the mobile wireless network IP based.
\end{abstract}

Keywords- Mobile wireless network; Unilateral TCPaccelerate; Parallel TCP-accelerate

\section{INTRODUCTION}

IP technology has become an important means of information transmission in wireless system, such as wireless mesh network, ad hoc network and so on, and the transmit control protocol called TCP, which is a connection oriented, reliable and end-to-end byte stream communication protocol, has been used widely for providing a continuous and realtime video and audio stream. However, the performance of the TCP protocol which is designed for the wired network orient has been reduced in the wireless network due to the diversity of the network, the complex of wireless channel, and the long-distance of data transmit, and it is now difficult to meet the requirements of the wireless network.

The paper analyzed the cause of the problem TCP faced in part II, and clarified the necessary of researching the TCP enhancement technology; in part III, some popular TCP enhancement algorithms have been summarized and concluded; two methods of TCP enhancement has been talked deeply in part IV, and the unilateral TCP-accelerate module and parallel TCP-accelerate module were designed and test in a prototype network and a $3 \mathrm{G}$ system; part V is a conclusion of the paper.

\section{The DisAdVANTAGE OF TCP IN WiRELESS NETWORK}

The reliable data stream provided by TCP protocol is by sequence confirm and packet retransmission mechanism, but the TCP window size and window management mechanism in TCP protocol cannot reflect the state of the network quickly when there are packets loss and delay in network path, and it leads to a significant decline of the throughput of TCP and cannot use of bandwidth efficient resulting the idle and waste of the bandwidth. There are three aspects could explain it:

First, the data rate is reduced by high error rate. There is a high bit error rate in wireless network which are highly susceptible to topography, weather, climate, human disturbance and other factors. TCP protocol will reduce the value of data transmit window which lowering of data transmission speed, and retransmit the packets, and start the corresponding congestion control when data loss happened caused by link error,.

Second, data recovery is slow caused by long propagation delay. The RTT (Round-Trip Time) of wireless channel is larger than the ground network, for example, the RTT of satellite link is even up to $560 \mathrm{~ms}$ [1]. TCP transmission control algorithm uses an end-to-end and closed-loop control mode based on the congestion window and the efficiency of the algorithm has a close relation with the feedback delay. Long propagation delay will make the congestion window grows and data recovery slowly and reduces the performance of network transmission.

Third, Asymmetric link makes a delay confirmation and excessive retransmission. Lots of wireless systems adopt asymmetric access, the uplink channel transmission rate tend to differ with down link channel transmission rate. In TCP protocol, the window size is adjusted and the packet rate is determined by the data-sender according to the ACK package returned from the data-receiver, but it always could not be adjusted on time due to the slowly ACK transfer rate caused by its queuing up after a large number of data packets and make congestion window grows slowly; furthermore, the ACK packet would be lost and a large number of unnecessary retransmission packets in the forward channel would emerge if backward channel produce congestion.

Therefore, researching on TCP enhancement technology is of great significance for increasing the service efficiency of TCP and enhancing the performance of wireless network.

\section{RELATED WORK OF TCP ENHANCEMENT TECHNOLOGIES}

Many researchers has invested a lot of time and energy on TCP enhancement technologies, and it now mainly 
formed four types of TCP enhancement technologies including TCP parameters optimization, the end-to-end TCP protocol modifications, unilateral TCP-accelerate technology and parallel TCP-accelerate technology.

\section{A. TCP parameters optimization}

The technology including B IC TCP, CUBIC TCP, FAST TCP, and H-TCP method, can reduce the time delay effects by adjusting and optimizing some TCP operation parameters while not changing the standard TCP protocol, but it is difficult to have greater improvement on TCP performance due to the increasing of packet loss, and must be optimized at both sender and receiver end.

\section{B. The end-to-end TCP protocol modifications}

The end-to-end protocol optimization scheme mainly aims at the control algorithm of TCP protocol. One way is to increase the congestion window initial value and increase data segment in the standard TCP from 1 to 4 . This method can shorten the duration of the slow start process and speed up the increase of the congestion window. While this method increases the risk of network congestion especially in the heterogeneous communication networks at the same time. Other ways, including TCP SACk, TCP Veges, TCP NewReno, TCP Westwood, TCP Peach and P-Start, etc, are to estimate the network condition through ACK return information and make it into a different stage of TCP. TCP SACk[2] method can improve the efficiency of the retransmission according to retransmit multiple missing data which is additional in the ACK information and including the disorderly data in a RTT period; TCP Veges[2] method calculates the optimal network throughput by round-trip time delay and then adjust the congestion window size according to the backlog of the amount of data in transmission pipeline which is get on the basis of the relationship between the optimal throughput and the sending rate; TCP Veno method is a further version of TCP Vegas, the method judge the congestion through the threshold value of the backlog of data; TCP NewReno[3] method considers that the quickly recover algorithm should not stop until multiple data lost happened; TCP Westwood[4] method get network resources according to the time of arrival of the ACK message; TCP Peach method adds suddenly start and fast recovery algorithms on the basis of congestion avoidance and fast retransmission algorithm; P-Start method is a grading mechanism of TCP slow Start[5].

Although the improved TCP protocol mentioned above can adapt to the characteristics of the wireless link, but they need to change the existing protocol a lot and are not convenient to engineering application limiting the flexibility of the wireless network.

\section{Unilateral and parallel TCP-accelerate methods}

Unilateral TCP-accelerate method, which needs be deployed at source end or destination end only, can keep the compatibility with the traditional TCP flow control mechanism and bring significant speedup of TCP. The intelligent flow control algorithms are introduced and the TCP/IP header fields are not modified in the Unilateral TCP- accelerate technology. ZETATCP [6] and SINFOR TCP [7] are two famous unilateral TCP-accelerate algorithms in the world.

Parallel TCP-accelerate method, which should be deployed in the application layer, can improve TCP performance by increasing the number of TCP connections and the collaboration between the TCP flows [8]. Parallel TCP, compared with the method of modified TCP protocol parameters, has a better resistance to jitter by the structure of multiple streams and has a good feasibility and expansibility by not modifying the standard TCP stack.

It could be concluded that, the method of TCP parameters optimization has limited ability in improving the performance of wireless network, and the method of end-toend TCP protocol modifications is not convenient to engineering application, but the unilateral TCP-accelerate and parallel TCP methods are simple and easy to implement, and can enhance the performance of TCP obviously at the wireless network.

\section{THE IMPLEMENTATION AND ANALYSIS OF TCP- ACCELERATE ALGORITHMS}

\section{A. design philosophy}

A unilateral TCP-accelerate module has been designed on the basis of ZETATCP algorithm and packed as a kernel module (*.ko), and it can be loaded dynamically according to the need of sender or receiver; a parallel TCP-accelerate dynamic link library (*.so file) in the application layer is designed also, and it provides friendly interfaces about IP address and port, send buffer size, and concurrent TCP streams number.

The basic idea of the unilateral TCP-accelerate algorithm mainly includes the improvement with optimization of bandwidth prediction, congestion control and slow start.

(1) Bandwidth prediction

The algorithm counts the available network bandwidth using the ACK message rate and the data size provided in ACK message. Assuming that the sender has received an ACK message at time $\boldsymbol{t}_{\boldsymbol{k}}$, and there are $\boldsymbol{d}_{\boldsymbol{k}}$ bytes have been received correctly, then the value of bandwidth sample can be calculated through the formula $\boldsymbol{b}_{\boldsymbol{k}}=\boldsymbol{d}_{\boldsymbol{k}} /\left(\boldsymbol{t}_{\boldsymbol{k}}-\boldsymbol{t}_{\boldsymbol{k}-1}\right), \boldsymbol{t}_{\boldsymbol{k}-1}$ is the time of ACK message received, we order that $\Delta \boldsymbol{t}_{\boldsymbol{k}}=\boldsymbol{t}_{\boldsymbol{k}}-\boldsymbol{t}_{\boldsymbol{k}-1}$, then $\boldsymbol{b}_{\boldsymbol{k}}=\boldsymbol{d}_{\boldsymbol{k}} / \Delta \boldsymbol{t}_{\boldsymbol{k}}$. If there were no other packets of data flow through this link, and these two ACK messages are triggered by data message in the same window, then the Statistical bandwidth sample is considered as the whole link bandwidth.

In order to obtain more reasonable value of available bandwidth, the following statistical algorithm is used:

First, make $\boldsymbol{b}_{\boldsymbol{k}}$ as the bandwidth sample of time $\boldsymbol{t}_{\boldsymbol{k}}, \hat{\boldsymbol{b}_{\boldsymbol{k}}}$ as the smooth value after low pass filter, and $\hat{\boldsymbol{a}_{\boldsymbol{k}}}$ is as the exponential filter parameters at time $\boldsymbol{t}_{\boldsymbol{k}}$, namely the value of $\hat{\boldsymbol{a}_{\boldsymbol{k}}}$ is time-varying. The value of $\hat{\boldsymbol{b}_{\boldsymbol{k}}}$ can be calculated by the following formula: 


$$
\hat{\boldsymbol{b}_{\boldsymbol{k}}}=\boldsymbol{a}_{\boldsymbol{k}} \hat{\boldsymbol{b}}_{\boldsymbol{k}^{-1}}+\left(1-\boldsymbol{a}_{\boldsymbol{k}}\right)\left(\frac{\boldsymbol{b}_{\boldsymbol{k}}+\boldsymbol{b}_{\boldsymbol{k}-1}}{2}\right)
$$

And,

$$
\hat{a_{k}}=\frac{2 \tau-\Delta t_{k}}{2 \tau+\Delta t_{k}}
$$

Parameter $1 / \tau$ is the cut-off frequency, only the data less than $1 / \tau$ is retained, parameter $\boldsymbol{a}_{\boldsymbol{k}}$ mainly depend on the parameters $\Delta \boldsymbol{t}_{\boldsymbol{k}}$ to offset the impact of uncertain interval. As can be seen from the formula, when time interval $\Delta \boldsymbol{t}_{\boldsymbol{k}}$ increase, the relationship between the new smooth value $\hat{\boldsymbol{b}_{\boldsymbol{k}}}$ and the smooth value $\hat{\boldsymbol{b}_{\boldsymbol{k}-1}}$ a moment before is weakened, and when time interval $\Delta \boldsymbol{t}_{\boldsymbol{k}}$ decrease, the relationship is strengthened.

It proves that the bandwidth value calculated by the method is more accurate than the bandwidth value calculated by the traditional method after long time testing.

(2) Congestion control

The congestion of network and the data rate can be determined by the bandwidth value obtained with above method. First of all, the bandwidth value is saved temporarily and sent after waiting for a RTT time; at the same time, the network bandwidth is updated and the data rate is adjusted according to the received ACK message. If there are no received ACK messages within a RTT time, the subsequent processing is determined by comparing the relationship between the received reduplicate ACK packet (dupACKS) and the current network bandwidth (cur_BW).

if dupACKS < cur_BW * rate ( setting rate, $0<$ rate $<1$ ), it considers that the data delay is caused by error of packet transmission and there is no network congestion, then the send window and the slow start threshold is updated by updating the degree of increasing bandwidth;

if dupACKS $>=$ cur_BW $*$ rate, it considers that the network congestion is similar with previous or worse in the time period, and the congestion avoidance algorithm is started.

(3) Slow start

The core idea of slow start algorithm is that the congestion window following the law of exponential growth when it is less than half of the slow start threshold, otherwise, the congestion window approaching congestion threshold gradually increases with the half of the difference of the congestion window and congestion threshold each time, and then entered the stage of congestion avoidance ${ }^{[9]}$.

The algorithm is shown in figure 1 , make the value of CWND equals to (CWND + SSTHRESH) / 2 when congestion window has grown to half value of SSTHRESH, and make the $\triangle \mathrm{CWND}$ which will be decreased with the increase of CWND equals to (SSTHRESH-CWND) / 2, the standard slow start algorithm which may produce excessive burst data flow is improved by the small increment of the injection data within the RTT period.

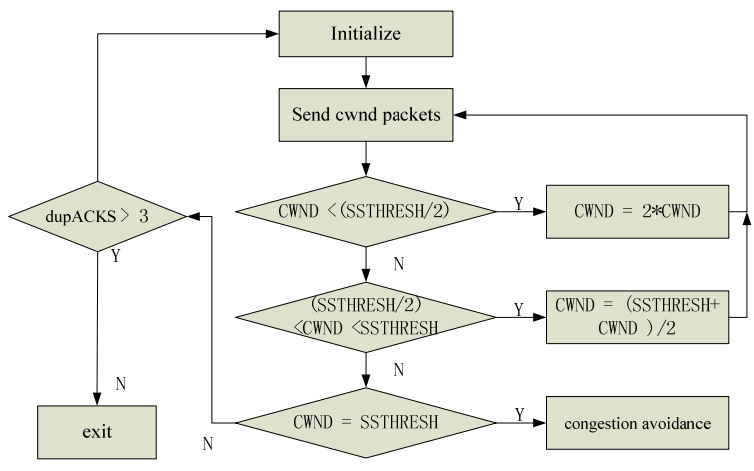

Figure 1. Slow start algorithm flow chart.

The Parallel TCP-accelerate module is realized in the application layer, the TCP SOCKET are created by the data sender (server) to listen a connection request from the client, and one TCP connection and a new thread are established when a connection request received, and every TCP connection can be configured as a type of competition or a type of Equality to send the information.

\section{B. The applying and analyzing of two TCP-accelerate modules}

A semi-physical wireless network simulated $802.11 \mathrm{n} \mathrm{Wi-}$ Fi environment is built to test the effects of the two TCPaccelerate modules. As shown in figure 2, the bandwidth in theory is 200 Mbps for upload and download each, and the real upload and download capacity of the network less than 60 Mbps and 140 Mbps on the condition of testing large file when no TCP-accelerate modules are imported. If the unilateral TCP-accelerate module is loaded, the average upload and download capacity improves obviously by all up to nearly 170 Mbps. If the parallel TCP strategy is used at the application layer, the data throughput will be smoother as the increase of the parallel TCP data flows, and it is meaning for real-time audio and video data transmission. There is one thing to note that the fairness of bandwidth allocation will be affected by increasing the parallel TCP flows when congestion is happened, the test has proved that, 3 to 5 parallel TCP flows are best for video transmission.

The unilateral TCP-accelerate module and parallel TCP dynamic link library are also used to a 3G wireless audio and video transmission system which has poorer channel environment than the semi-physical wireless network.

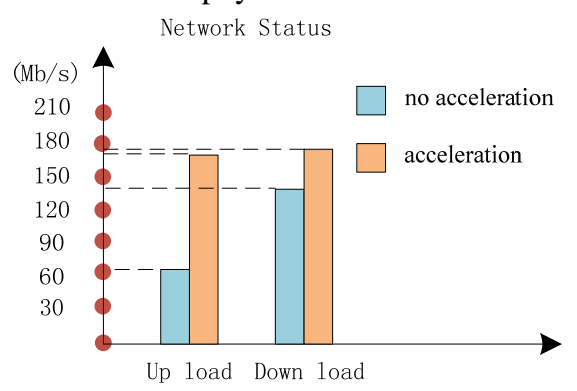

Figure 2. Test in the semi-physical wireless network. 


\section{CONCLUSION}

The cause of TCP performance degradation in wireless network is analyzed and the popular TCP enhancement technologies are expounded fully in the paper, a unilateral TCP-accelerate module and a parallel TCP-accelerate module are designed and used in a test network and a 3G video and audio transmission system. It proved that these two modules are easy to deploy and have a good effect on improving TCP performance. But we should also know that they are not always perfect, as mentioned above, the performance will be descended if there are too many parallel TCP flows; and we do have a reason to worry about the practical performance of them for not yet test in a real dynamic and large wireless network.

\section{REFERENCES}

[1] Jian Wang, Hai-tao Wang, "TCP Performance Enhancement in Satellite Communication System”, SCIENCE TECHNOLOGY AND ENGINEERING, 2009,6: 3148-3152
[2] Hai Jiang etc, "TCP SACK and TCP Vegas Performance in Wireless Ad Hoc Networks", JOURNAL OF CIRCUITS AND SYSTEMS, 2001, 6(1).

[3] Wei Sun etc, "Steady State Throughput Modeling of TCP NewReno", JOURNAL OF COMPUTER RESEARCH AND DEVELOPMENT, 2010, 47(3).

[4] Hua Peng, Ya-ping Deng, "Enhancement based on TCP westwood", COMPUTER ENGINEERING AND DESIGN, 2006, 27(21).

[5] Xiao-heng Deng, "P-Start: a Phase-Divided TCP Slow Start Mechanism”, MINI-MICRO SYSTEMS, 2005, 26(10).

[6] AppEx Networks, ZetaTCP White Paper, http://www.appexnetworks.com/white-papers/ZetaTCP.pdf

[7] SANGFOR INC, Q-Series-Whitepaper, http://www.sangfor.com/product_doc/whitepaper/Q-SeriesWhitepaper.pdf

[8] Yong-zhe Gui, Jin-yu Zhang, "Data transfer technology based on overlay multi-pathing and parallel TCP:, JOURNAL OF COMPUTER APPLICATIONS, 2010, 30(5) : 5-8.

[9] Liang Fan, "A slow start strategy based on the factor of proportional factor", JOURNAL OF SICHUAN UNIVERSITY(NATURAL SCIENCE EDITION), 2009, 46(5). 